\title{
The TP53 mutation rate differs in breast cancers that arise in women with high or low mammographic density
}

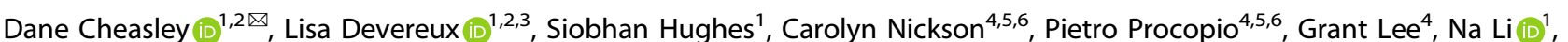 \\ Vicki Pridmore ${ }^{7}$, Kenneth Elder ${ }^{8,9,10}$, G. Bruce Mann ${ }^{2,8,9}$, Tanjina Kader ${ }^{10}{ }^{1,2}$, Simone M. Rowley ${ }^{1}$, Stephen B. Fox ${ }^{11}$, David Byrne iD $^{11}$, \\ Hugo Saunders ${ }^{1}$, Kenji M. Fujihara (D) ${ }^{1}$, Belle Lim ${ }^{1,12}$, Kylie L. Gorringe ${ }^{2,13,14}$ and lan G. Campbell (iD) ${ }^{1,2,14}$
}

Mammographic density (MD) influences breast cancer risk, but how this is mediated is unknown. Molecular differences between breast cancers arising in the context of the lowest and highest quintiles of mammographic density may identify the mechanism through which MD drives breast cancer development. Women diagnosed with invasive or in situ breast cancer where MD measurement was also available $(n=842)$ were identified from the Lifepool cohort of $>54,000$ women participating in populationbased mammographic screening. This group included 142 carcinomas in the lowest quintile of MD and 119 carcinomas in the highest quintile. Clinico-pathological and family history information were recorded. Tumor DNA was collected where available $(n=56)$ and sequenced for breast cancer predisposition and driver gene mutations, including copy number alterations. Compared to carcinomas from low-MD breasts, those from high-MD breasts were significantly associated with a younger age at diagnosis and features associated with poor prognosis. Low- and high-MD carcinomas matched for grade, histological subtype, and hormone receptor status were compared for somatic genetic features. Low-MD carcinomas had a significantly increased frequency of TP53 mutations, higher homologous recombination deficiency, higher fraction of the genome altered, and more copy number gains on chromosome $1 \mathrm{q}$ and losses on $17 \mathrm{p}$. While high-MD carcinomas showed enrichment of tumor-infiltrating lymphocytes in the stroma. The data demonstrate that when tumors were matched for confounding clinico-pathological features, a proportion in the lowest quintile of MD appear biologically distinct, reflective of microenvironment differences between the lowest and highest quintiles of MD.

npj Breast Cancer (2020)6:34; https://doi.org/10.1038/s41523-020-00176-7

\section{INTRODUCTION}

High mammographic density (MD) is associated with a significant increase in breast cancer risk ${ }^{1,2}$, with those in the highest quintile of MD having 4-6-fold increased risk compared to women in the lowest quintile of $\mathrm{MD}^{3}$. Within population-based breast mammographic screening programs, women with high MD experience an increased rate of interval breast cancer diagnosis ${ }^{4,5}$.

Few studies have explored the molecular landscape of cancers arising in low and high MD, with the majority of studies focusing on altered expression of a few molecules involved in extracellular matrix formation ${ }^{6-10}$ or single-nucleotide polymorphisms (SNPs) associated with both MD and breast cancer risk ${ }^{11-13}$. However, the additive effects of these common SNPs explain only a small percentage $(<5 \%)$ of total MD variance with the remainder possibly attributed to unknown genes. Studies have investigated strong breast cancer susceptibility genes, BRCA1 and BRCA2, and show no significant link with $\mathrm{MD}^{14,15}$.

While large repositories of genomic data are available for breast cancer, these data are seldom linked to MD scoring and consequently it is unknown if the molecular mechanisms of breast cancer development differ in dense versus non-dense breasts. An understanding of the underlying biological reasons why women with dense breasts are at a higher risk for developing breast cancer may identify opportunities to reduce that risk. The reason for the association between $\mathrm{MD}$ and breast cancer risk is currently unknown, although it is reasonable to presume that it reflects differences in the microenvironment. Consequently, if the drivers of breast cancer development in low- and high MD are different this might also result in breast cancers with divergent molecular profiles and provide evidence of a direct link between MD and breast cancer development. Therefore, the aim of this study was to determine the biological relationship of breast cancers arising in the lowest and highest quintiles of MD through somatic genomic analyses of a large cohort of well annotated breast cancers.

\section{RESULTS}

Dense breasts exhibit a more aggressive phenotype

A total of 842 Lifepool participants were identified as having being diagnosed with a breast carcinoma (either invasive or in situ) where a mammogram preceding the diagnosis was available for calculation of an MD score. Among the 670 invasive breast cancers, 142 and 119 were categorized as arising in breasts of the

\footnotetext{
${ }^{1}$ Cancer Genetics Laboratory, Peter MacCallum Cancer Centre, Melbourne, VIC, Australia. ${ }^{2}$ Sir Peter MacCallum Department of Oncology, University of Melbourne, Melbourne, VIC, Australia. ${ }^{3}$ Lifepool, Peter MacCallum Cancer Centre, Melbourne, VIC, Australia. ${ }^{4}$ Centre for Epidemiology and Biostatistics, Melbourne School of Population and Global Health, University of Melbourne, Melbourne, VIC, Australia. ${ }^{5}$ Cancer Research Division, Cancer Council NSW, Sydney, NSW, Australia. ${ }^{6}$ Sydney School of Public Health, University of Sydney, Sydney, NSW, Australia. ${ }^{7}$ BreastScreen Victoria, Carlton South, VIC, Australia. ${ }^{8}$ Department of Surgery, University of Melbourne, Melbourne, VIC, Australia. ${ }^{9}$ The Royal Melbourne and Royal Women's Hospitals, Parkville, VIC, Australia. ${ }^{10}$ The Edinburgh Breast Unit, Western General Hospital, Edinburgh, UK. ${ }^{11}$ Department of Pathology, Peter MacCallum Cancer Centre, and University of Melbourne, Melbourne, VIC, Australia. ${ }^{12}$ Drug Discovery Biology, Monash Institute of Pharmaceutical Sciences, Monash University, Parkville, VIC, Australia. ${ }^{13}$ Cancer Genetics and Genomics Program, Peter MacCallum Cancer Centre, Melbourne, VIC, Australia. ${ }^{14}$ These authors contributed equally: Kylie L. Gorringe, lan G. Campbell. ${ }^{凶}$ email: dane.cheasley@petermac.org
} 
Table 1. Clinico-pathological features of breast cancers diagnosed in the lowest and highest quintiles of mammographic density.

\begin{tabular}{|c|c|c|c|}
\hline Characteristics & $\begin{array}{l}\text { Lowest } \\
\text { quintile }\end{array}$ & $\begin{array}{l}\text { Highest } \\
\text { quintile }\end{array}$ & $P$ value \\
\hline Invasive & 142 & 119 & \\
\hline \multicolumn{4}{|l|}{ Age at diagnosis } \\
\hline Mean \pm SD & $64.5 \pm 7.0$ & $61.5 \pm 7.5$ & $0.0007^{\mathrm{a}}$ \\
\hline Median & 65.5 & 61.4 & \\
\hline Range & $47-88$ & $43-81$ & \\
\hline \multicolumn{4}{|l|}{ Screening timing } \\
\hline Lapsed screener & $2(1 \%)$ & $1(1 \%)$ & $0.0006^{b}$ \\
\hline Interval cancer & $10(7 \%)$ & 27 (23\%) & \\
\hline Screen detected & $130(92 \%)$ & 91 (76\%) & \\
\hline \multicolumn{4}{|l|}{ Tumor size } \\
\hline$<20 \mathrm{~mm}$ & $80(60 \%)$ & $57(53 \%)$ & $0.3966^{b}$ \\
\hline $20-49 \mathrm{~mm}$ & $39(30 \%)$ & $41(38 \%)$ & \\
\hline$\geq 50 \mathrm{~mm}$ & $13(10 \%)$ & $10(9 \%)$ & \\
\hline NA & 10 & 11 & \\
\hline \multicolumn{4}{|l|}{ Intrinsic subtype } \\
\hline TNBC & $10(7 \%)$ & $6(6 \%)$ & $0.9450^{c}$ \\
\hline ER-, Her2+ & $2(1 \%)$ & $2(2 \%)$ & \\
\hline Luminal Her2+ & $8(6 \%)$ & $7(7 \%)$ & \\
\hline Luminal & $114(86 \%)$ & $90(87 \%)$ & \\
\hline NA & 8 & 14 & \\
\hline \multicolumn{4}{|c|}{ Invasive cancer histology subtype } \\
\hline Ductal & $117(82 \%)$ & 91 (76\%) & $0.3523^{b}$ \\
\hline Lobular & $7(5 \%)$ & $11(10 \%)$ & \\
\hline Other invasive & $18(13 \%)$ & 17 (14\%) & \\
\hline \multicolumn{4}{|l|}{ Tumor grade } \\
\hline G1 & $34(26 \%)$ & $31(29 \%)$ & $0.4003^{b}$ \\
\hline G2 & $62(47 \%)$ & 55 (51\%) & \\
\hline G3 & $36(27 \%)$ & $21(20 \%)$ & \\
\hline NA & 10 & 12 & \\
\hline \multicolumn{4}{|l|}{ Nodal status } \\
\hline Positive & $22(21 \%)$ & $19(20 \%)$ & $>0.9999^{\mathrm{b}}$ \\
\hline Negative & $82(79 \%)$ & $75(80 \%)$ & \\
\hline NA & 38 & 25 & \\
\hline \multicolumn{4}{|c|}{ Proliferation index (Ki67) } \\
\hline High ( $\geq 15 \%)$ & $19(25 \%)$ & 23 (35\%) & $0.1976^{b}$ \\
\hline Low $(<15 \%)$ & $58(75 \%)$ & $42(65 \%)$ & \\
\hline NA & 65 & 54 & \\
\hline \multicolumn{4}{|c|}{ First-degree relatives with breast cancer } \\
\hline Yes & 39 (27\%) & 46 (39\%) & $0.0637^{b}$ \\
\hline No & $103(73 \%)$ & $73(61 \%)$ & \\
\hline \multicolumn{4}{|c|}{ Strong family history of breast cancer } \\
\hline Yes & $11(8 \%)$ & $20(17 \%)$ & $0.0336^{b}$ \\
\hline No & $131(92 \%)$ & $99(83 \%)$ & \\
\hline
\end{tabular}

lowest and highest MD quintiles, respectively (Table 1). Women diagnosed with an invasive breast cancer in the highest MD quintile had a significantly lower mean age at diagnosis (61.5 [range 43-81]) compared with the lower quintile (64.5 [range 47-88]) $(p=0.0007)$ (Table 1). Invasive breast cancers in the highest quintile were significantly more likely to be interval breast cancer diagnoses $(p=0.0006)$ and a self-reported strong family history of breast cancer $(p=0.0336)$ (Table 1). The intrinsic subtype distribution (according to St Gallen classification) ${ }^{16}$ (Table 1, Supplementary Table 1), invasive cancer histology subtype distribution, tumor grade, nodal involvement, and tumor proliferation (as measured by Ki67 staining) were not statistically different between cancers arising in the lowest versus highest MD quintiles (Table 1).

Among the 172 in situ breast cancers, 31 and 58 were categorized as arising in breasts of the lowest and highest MD quintiles, respectively. Age at diagnosis, screening timing, tumor size, grade, histology, estrogen receptor status, and family history were compared and showed no significant differences (Supplementary Table 2).

\section{Differences in somatic mutation profile}

Targeted gene exon sequencing of 39 known breast cancer driver and hereditary predisposition genes, including 28 DNA repair genes (Supplementary Table 3), was performed on tumor DNA from all available lowest quintile $(n=29)$ and highest quintile breast cancers $(n=27)$, excluding in situ tumors (Supplementary Dataset). The cancers in each quintile were similar in age and grade (Supplementary Table 4).

Collectively, there were no significant differences in the somatic mutation frequency per $\mathrm{Mb}$ per tumor in the lowest MD compared with the highest MD $(0.78$ [range $0.21-1.91$ ] versus 0.83 [range $0.11-4.55], p=0.459)$, and this was not different when comparing within the luminal $(p=0.696)$, ductal $(p=0.403)$, and combined luminal/ductal $(p=0.461)$ subtypes (Supplementary Fig. 1). Luminal/ductal cancers were prioritized in the sub-analysis to remove the potential bias associated with lobular breast cancer diagnosis which can be masked in dense breasts ${ }^{17}$. The frequency of somatic mutations in each of the most commonly mutated breast cancer driver genes (PIK3CA, MAP3K, KMT2C, TP53, GATA3, $C D H 1$, and $(B F B)$ were similar between the two groups with the exception of TP53 (Table 2). The frequency of somatic mutations in breast cancer driver genes between the lowest and highest quintile was also compared within triple-negative breast cancers and lobular breast cancers showing no significant differences but the number of cancers compared was low (Supplementary Table 5).

A limitation to the TP53 mutation finding is that it is based on small numbers, and tumor sequencing was prioritized to samples within the highest and lowest quintiles of MD, and as such the TP53 mutation frequency in the intermediate quintiles was unknown. To address this issue, immunohistochemistry (IHC) for p53 expression was performed on tissue microarrays (TMAs) prepared for 180 breast cancers that encompassed the entire quintile range of $\mathrm{MD}$. Cases within each quintile of $\mathrm{MD}$ were determined to be either mutant (p53 absent, overexpression, or cytoplasmic staining in cancer cells) or wild type for p53. Of the cases analyzed by $\mathrm{IHC}, 80$ overlapped with those cases that had targeted sequencing, and the concordance of TP53 status with IHC was 79/80. The discordant case (Lifepool ID 13198, Supplementary Dataset) which is a quintile 5 metaplastic TNBC was due to the $>80 \%$ stromal cells within the two independent tumor cores. Among the entire cohort (Fig. 1a), luminal (Fig. 1b) or luminal/ ductal subtypes (Fig. 1c), p53 mutations were observed at a significantly higher frequency in breast cancers from the lower quintile compared to the highest quintile MD group ( $24 \%$ versus $6 \%, p=0.0499 ; 22 \%$ versus $0 \%, p=0.0058$; and $21 \%$ versus $0 \%$, $p=0.0390$, respectively).

Clinico-pathological and genetic features were compared between TP53 mutant versus TP53 wild-type cases in the lowest quintile of MD. TP53 mutant cases were significantly larger $(p=0.0354)$ and were of higher grade $(p=0.0028)$ compared to 
Table 2. Somatic driver mutation profile in breast cancers diagnosed in the lowest and lowest quintiles of mammographic density.

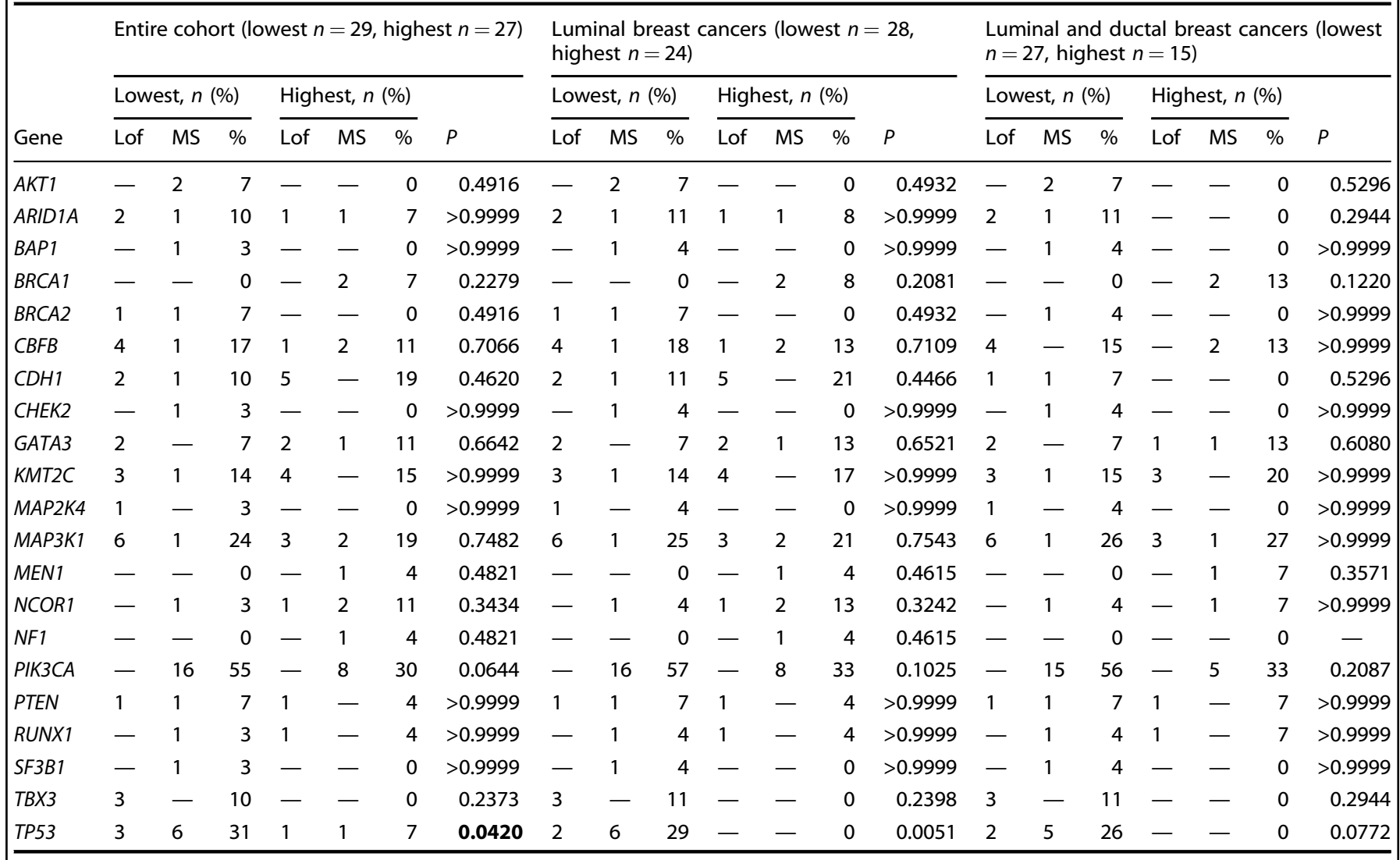

A two-tailed $p$ value was calculated. Bold $p$ values highlight somatic mutations that were significantly different between low- and high-MD breast cancers.
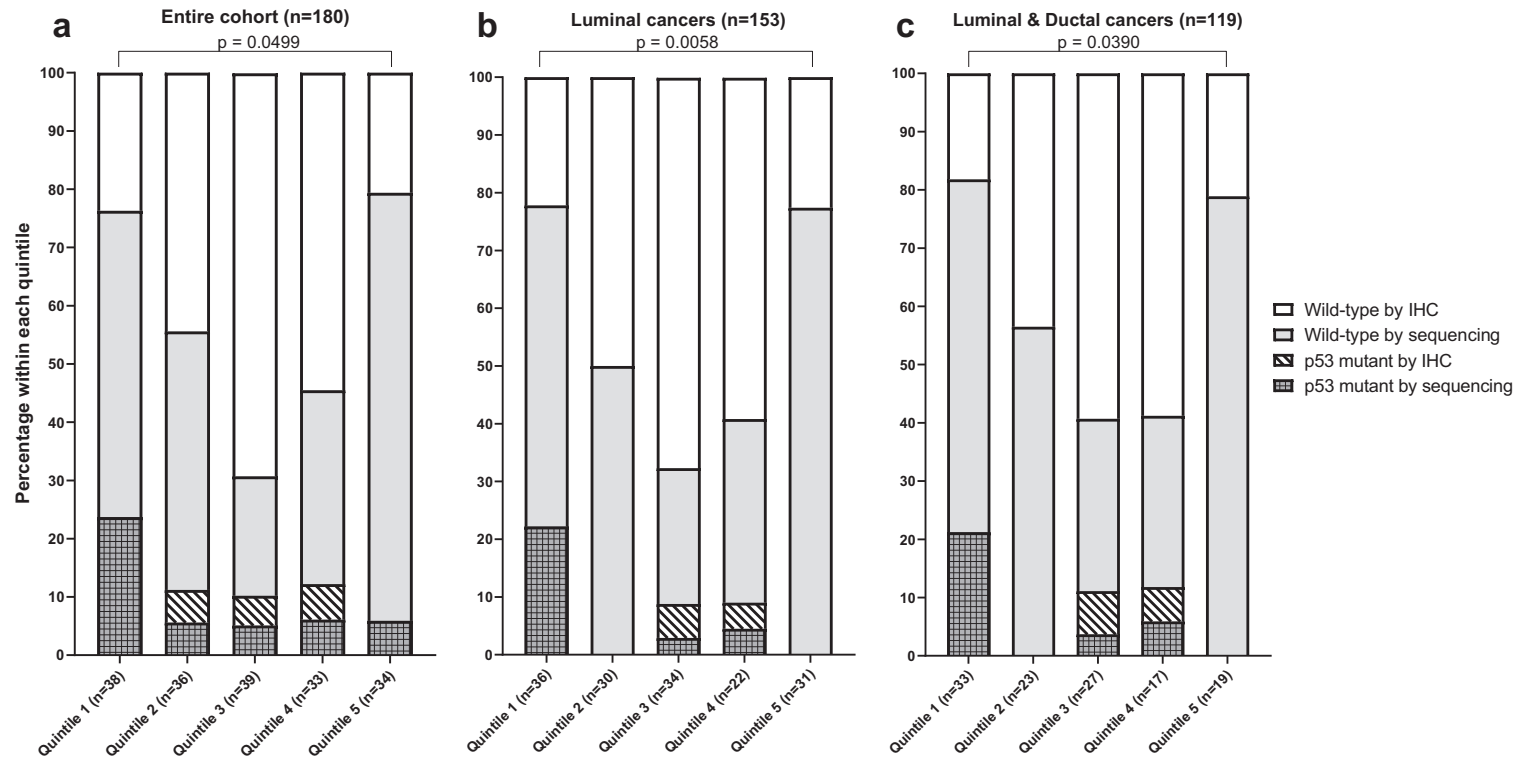

Fig. 1 p53 mutation analysis in breast carcinomas arising in all quintiles of mammographic density. Barplots showing the percentage of cases within each quintile of MD that were either mutant or wild type for p53, scored for either $\mathbf{a}$ the entire breast cancer cohort, $\mathbf{b}$ luminal cancers only, and c combined luminal subtype and ductal histology cancers.

TP53 wild-type cancers (Supplementary Table 6). No significant differences in somatic driver gene mutations or DNA repair gene mutations (excluding TP53) were observed (Supplementary Table 7).
Somatic copy number aberration differences

Analysis of genome-wide copy number changes showed regions of copy number aberration that were significantly different in frequency between the lowest and highest quintiles of $M D$, with 


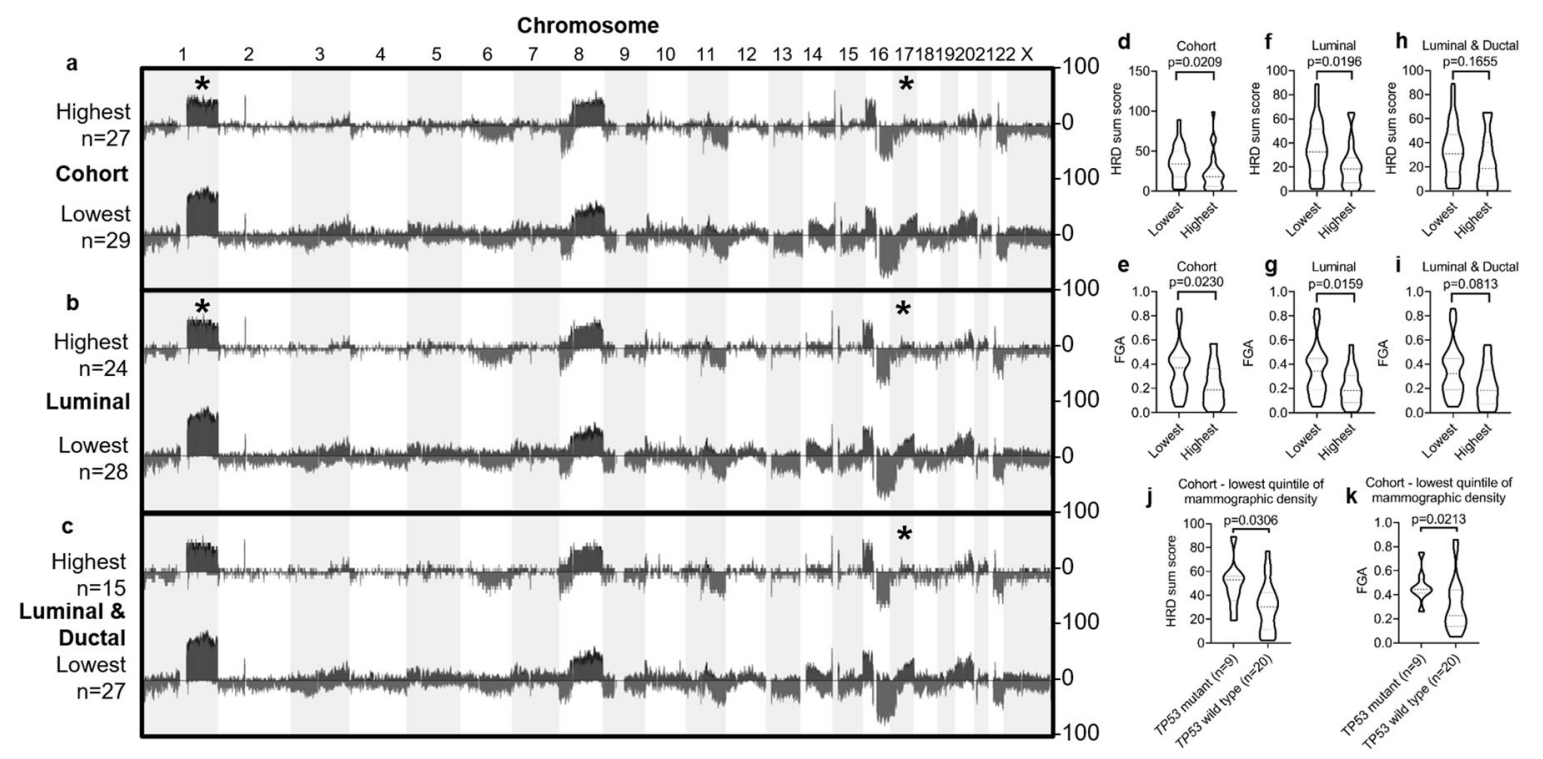

Fig. 2 Copy number alterations in low and high mammographic dense breast cancers. Copy number aberrations are shown for 25 high and 30 low-MD breast cancers as the frequency of copy number changes in a the entire cohort, $\mathbf{b}$ luminal subtype, and $\mathbf{c}$ combined luminal/ductal subtype. The chromosome region highlighted with an asterisk represents a significant copy change between the two cohorts (thresholds of $p<0.05$ and at least 25\% frequency difference). Copy number profiles were used to generate a homologous recombination deficiency (HRD) sum score for $\mathbf{d}$ the entire cohort, e luminal subtype, and $\mathbf{f}$ combined luminal/ductal subtype. Copy number profiles were used to generate a fraction of the genome altered (FGA) score for $\mathbf{g}$ the entire cohort, $\mathbf{h}$ luminal subtype, and $\mathbf{i}$ combined luminal/ductal subtype. Both HRD (j) and FGA (k) were compared between TP53 mutant and wild-type carcinomas in the lowest quintile of MD. Mann-Whitney test was applied to HRD and FGA violin plots.

the specific loci differences shown in Supplementary Table 8. Copy number gains on chromosome 1q (1q32.2 and 1q41) and loss of $17 p(17 p 12$ and 17p13.2-13.3) were enriched in the lowest MD quintile cases compared to the highest MD quintile cases across the entire cohort (Fig. 2a) or if stratified by luminal subtype (Fig. 2b). Copy number loss on $17 p$ was also significantly higher among the lowest MD quintile cancers when analysis was restricted to luminal cancers of ductal histology (Fig. 2c). Nine out of the ten cases with $17 \mathrm{p} \mathrm{LOH}$ carried a TP53 mutation.

The mean homologous recombination deficiency (HRD) sum score was significantly higher in the low-MD cancers within the entire cohort (mean score 35.2 versus 23.4, $p=0.0209$, Fig. 2d) and luminal subtype (mean score 35.0 versus 21.1, $p=0.0196$, Fig. 2e), but not combined luminal-ductal cancers (mean score 34.3 versus $24.2, p=0.1655$, Fig. $2 f$ ). The mean fraction of the genome altered (FGA) score was also significantly higher in the low-MD cancers within the entire cohort (mean score 0.36 versus $0.23, p=0.0230$, Fig. $2 \mathrm{~g}$ ) and luminal subtype (mean score 0.35 versus $0.21, p=0.0159$, Fig. $2 \mathrm{~h}$ ), but not combined luminalductal cancers (mean score 0.35 versus $0.22, p=0.0813$, Fig. $2 \mathrm{i}$ ). TP53 mutant cancers were compared to TP53 wild-type cancers in the lowest quintile of $\mathrm{MD}$ and showed a significantly higher mean HRD sum score (mean score 49.0 versus $30.2, p=0.0306$, Fig. 2j) and FGA (mean score 0.47 versus $0.31, p=0.0213$, Fig. $2 \mathrm{k}$ ).

To investigate the genomic basis of the difference in HRD and FGA between the low- and high-MD cancers, 28 genes involved in DNA repair (Supplementary Table 3 ) were sequenced in the breast cancers. Excluding TP53, the frequency of somatic mutations in individual genes nor the sum of somatic mutations in all DNA repair genes differed significantly between low-MD and high-MD cancers (Supplementary Table 9). Similarly, HRD high cancers (scores $\geq 42$ ) showed no significant difference in the frequency of mutations in homologous recombination-linked genes compared to HRD low cancers (scores < 42) (Supplementary Table 9).

Of the 56 breast cancers sequenced, 12 (5 low MD and 7 high MD) had no detectable somatic driver gene mutations, but did harbor reliable HRD and FGA data indicating they were not poor samples due to low tumor purity.

Differences in stromal tumor-infiltrating lymphocytes (TILs)

Assessing an average percentage of stromal TILs/total area of intra-tumoral stromal area was performed and showed a significantly higher percentage of stromal TILs in the highest quintile compared to the lowest quintile of MD in the cohort as whole (Fig. $3 a, p=0.0009$ ) or when stratified by luminal subtype (Fig. $3 b, p=0.0018$ ) or luminal/ductal subtype (Fig. $3 c, p=0.0057$ ). Additionally, the percentage of stromal TILs in TP53 wild type and TP53 mutant low-MD carcinomas were not significantly different in the cohort (Fig. $3 \mathrm{~d}, p=0.2371$ ), luminal subtype (Fig. 3e, $p=$ 0.3862 ), or luminal/ductal subtype (Fig. $3 f, p=0.2294$ ); however, the overall number of TP53 mutant cases is small and these data need to be interpreted cautiously.

\section{DISCUSSION}

Previous clinico-pathological analyses have shown that cancers arising in women with breasts of high versus low MD are very similar, but none have explored if this extends to the molecular genetic characteristics. In this study we report the largest and most detailed somatic genetic analysis of these two cohorts to gain insights into the mechanism of cancer predisposition associated with MD. The clinico-pathological features of our cohort are largely consistent with data reported in other studies ${ }^{18,19}$ showing cancers arising in the highest quintiles were more likely to be associated with younger age at diagnosis, an interval cancer diagnosis and a strong family history of breast cancer $^{20-22}$.

The genomic data suggest that breast cancers arising in the highest and lowest quintiles of MD are very similar with respect to copy number profiles and overall frequency and spectrum of driver gene mutations but with the notable exception of TP53. The low frequency of TP53 mutations observed in high-MD cancers 
a

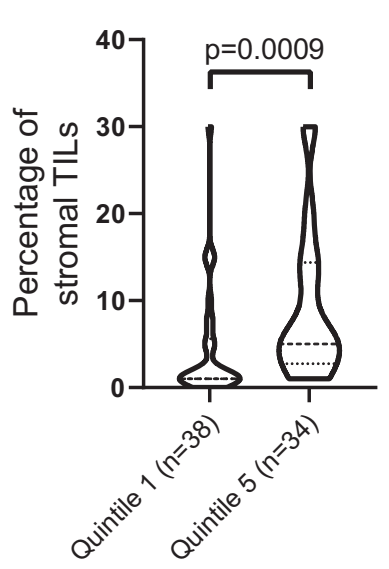

d
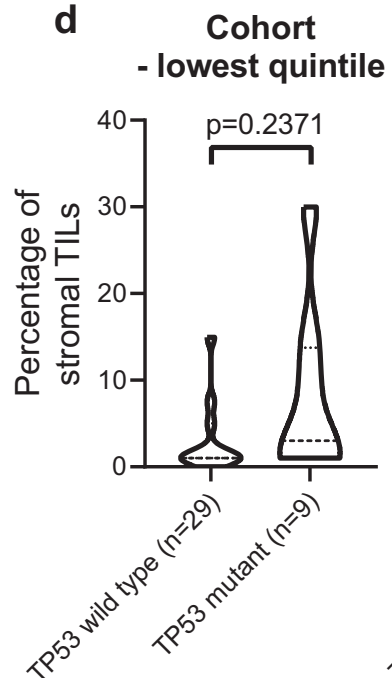

b

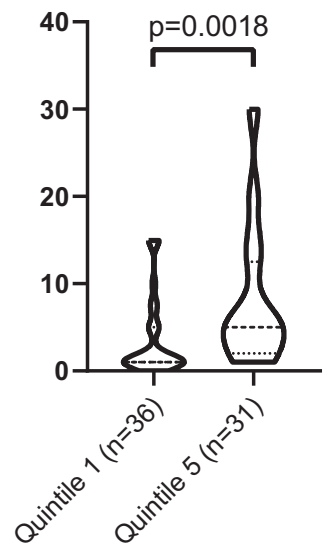

e

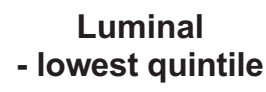

(15-10
C Luminal \& Ductal

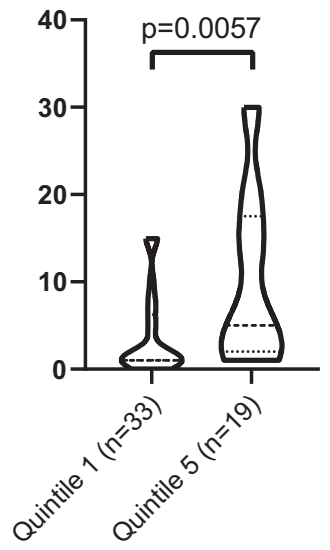

f Luminal \& ductal

- lowest quintile

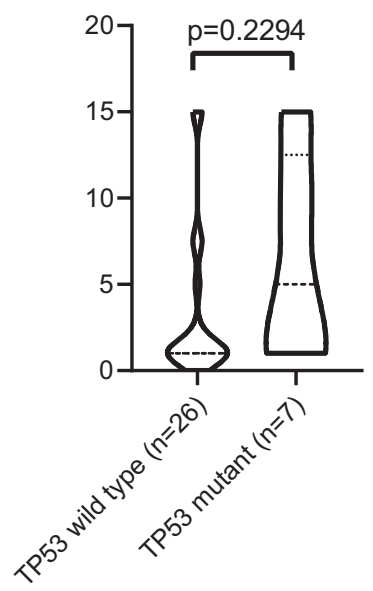

Fig. 3 Comparing stromal tumor-infiltrating lymphocytes. Violin plots showing the percentage of stromal TILs in the tumor area for the full $\mathrm{H} \& \mathrm{E}$-stained section. Percentage is scored comparing breast cancers in the lowest and highest quintiles of MD within a the entire breast cancer cohort, b luminal cancers only, $\mathbf{c}$ and combined luminal subtype and ductal histology cancers. Assessing breast cancers in the lowest quintile of MD only, the percentage of stromal TILS was scored comparing TP53 wild type and TP53 mutant breast cancers within $\mathbf{d}$ the entire breast cancer cohort, e luminal cancers only, $\mathbf{f}$ and combined luminal subtype and ductal histology cancers. Mann-Whitney test was applied.

may reflect the cancer growth promoting influence of the rich stromal microenvironment of dense breasts precluding the need for mutation of this strong somatic driver gene. One potential cancer-promoting factor in high-MD breast is tumor-promoting immune cells that have been shown in humans to be more prevalent in high- versus low-MD breasts ${ }^{23}$. Consistent with this we observed that among the entire cohort there was enrichment of TILs in the stroma of high-MD cancers compared to low-MD cancers. However, among the low-MD tumors where there were sufficient cases with TP53 mutations, there was no difference in the percentage of stromal TILs between TP53 wild type and mutant cases, suggesting that the immune microenvironment does not directly influence acquisition of TP53 mutations.

The frequency of chromosome $17 p$ loss was increased in the low-MD compared to high-MD cancers, even after adjustment for tumor histology. This pattern of genomic alteration is consistent with the TP53 mutation data in low-MD cancers. TP53 is located on $17 \mathrm{p} 13.1$ and is considered to be the major driver of 17p13.2-p13.3 loss in breast cancer, reflecting a reduced dosage of linked tumor suppressor genes within these regions ${ }^{24,25}$. The high HRD and FGA scores observed in low- compared to high-MD cancers appears largely driven by TP53 mutant cases, consistent with previous observations from an analysis of pan-cancer TCGA data showing higher HRD and FGA in TP53-mutated tumors ${ }^{26,27 .}$

There are still many gaps in the understanding of cellular and molecular mechanisms underlying the strong association of MD with breast cancer predisposition. While this study is the most comprehensive molecular analysis of low- and high-MD breast cancers, the overall numbers are small and these data need to be interpreted cautiously and will require validation in larger independent cohorts. Further insights might be gained by using additional image features of the dense tissue, and this may be the subject of future work.

\section{METHODS}

Study cohort

Subjects were recruited from the Lifepool study (www.lifepool.org) which is a prospective cohort of women participating in population-based mammographic screening. The women were cancer-free at the time of recruitment. Epidemiological and mammographic screening data were available for all study subjects as reported previously ${ }^{28}$. Cancer incidence 
was determined by linkage to the Victorian Cancer Registry and BreastScreen Victoria. Pathology assessment such as hormone receptor status, Ki67, and tumor grade was extracted from diagnostic pathology reports where available. A strong family history of breast cancer was defined according to the Australian referral guidelines for breast cancer risk assessment (www.eviq.org.au) as either three first- or second-degree relatives with breast cancer; two first- or second-degree relatives with breast cancer (one diagnosed $<50$ years); male breast cancer any age; or ovarian cancer any age. All participants in this study gave written informed consent and research was approved by the Peter MacCallum Cancer Centre Human Ethics Committee under protocol \#0966. All data are available as described in the Data Availability statement ${ }^{29}$

\section{Automated measurement of breast density}

Breast density measurements were attained from digital mammograms using AutoDensity ${ }^{30,31}$, which identifies the breast area in the digital mammogram (breast segmentation) and then classifies breast density by identifying distinctly white areas to be classified as "dense" (breast density segmentation). AutoDensity has been validated against Cumulus measurements, showing similar performances between the methods in terms of cancer risk, risk of interval breast cancers, and identifying the extreme quintiles of $M D^{32}$. For this study, percent $M D$ scores were generated from the most recent digital mammogram prior to breast tumor diagnosis. Scores were generated from the contralateral breast to cancer diagnosis and scores were adjusted for age at diagnosis.

\section{Tumor DNA extraction}

For formalin-fixed paraffin-embedded (FFPE) tumors, a representative haematoxylin and eosin (H\&E)-stained section was prepared and used as a template to needle-point micro-dissect cancer cells from subsequent 10$\mu \mathrm{m}$-thick-stained sections. DNA was extracted using the QIAamp DNA FFPE Tissue Kit (Qiagen, Valencia, CA, USA) and quantified using the Qubit dsDNA high-sensitivity assay kit (Thermo Fisher Scientific, Scoresby, Victoria Australia).

\section{Targeted sequencing and mutation detection}

Targeted sequencing of tumor DNA was performed using a SureSelect XT Custom Panel (Agilent Technologies, Santa Clara, CA), targeting all exons and intron-exon boundaries of 13 hereditary breast and ovarian cancer genes, 28 DNA repair genes, and 27 genes commonly somatically mutated in breast cancer ${ }^{28,33}$ (Supplementary Table 1). Library preparation was performed from an input of $300 \mathrm{ng}$ of tumor DNA using the KAPA Hyper Prep Kit (Kapa Biosystems, Wilmington, MA, USA). Sequencing of target-enriched DNA libraries was performed using the Illumina NextSeq500 generating paired-end 75 bp sequence reads. Somatic mutations in the tumor-sequencing data were identified by removing previously available germline variant data for $\mathrm{HBOC}$ panel genes, and where this information was not available, by applying the following filters: Transcript 1 ; quality $\geq 100$; read depth $\geq 20$; variants identified by at least two variant callers; minimum variant allele proportion $\geq 0.2$; and minor allele frequency present at $\leq 0.0001$ in the ExAc and GnomAD genomes database. Germline validation of genetic variants observed in tumors was performed on DNA extracted from either participant peripheral blood or saliva where available. Libraries were prepared using a custom-designed HaloPlex Targeted Enrichment Assay (Agilent Technologies, Santa Clara, CA) ${ }^{34-36}$. The variant data were filtered for loss-of-function mutations (defined as nonsense or frameshift or essential splice site mutations) and missense mutations that were classified as known pathogenic in ClinVar. Raw sequencing data are deposited in the NCBI Sequence Read Archive ${ }^{37}$.

\section{Genome-wide copy number analysis}

Off-target sequencing reads from the tumor-sequencing data were used to generate genome-wide copy number data for 29 lowest quintile and 25 highest quintile breast cancers using Copywrite $\mathrm{R}^{38}$, utilizing a normal lymphocyte DNA control run in the same sequencing batch for the normalization baseline (NA12878, Coriell Institute). Data were imported into NEXUS Copy Number ${ }^{\text {TM }}$ (software v8.0 with build version 9169, BioDiscovery Inc.), segmented using a FASST2 segmentation algorithm, and visualized. Comparisons between groups were made using Nexus applying thresholds of $p<0.05$ and at least $25 \%$ frequency difference. Within the Nexus software package gains were defined as $\log _{2}$ ratio $>0.3$ and losses at $<-0.3$. High copy number gains were called if the $\log _{2}$ ratio was $>1.2$ and homozygous deletions $<-1.2$.

\section{HRD and FGA scoring}

Using the genome-wide copy number data, an HRD sum score ${ }^{39}$ was calculated for each tumor by summing the individual scores for telomeric allelic imbalances, large-scale state transitions, and loss-of-heterozygosity across all chromosomes ${ }^{40,41}$. The FGA was the number of bases affected by copy number change for each chromosome divided by the total base pair size of that chromosome and then averaged across all chromosomes ${ }^{42}$.

\section{p53 IHC and scoring}

TMAs were constructed for Lifepool breast tumors with two independent $0.6 \mathrm{~mm}$ cores from each FFPE tumor tissue block. Sections were cut $(4-\mu \mathrm{m}-$ thick) and stained for p53 (clone DO-7; Leica Biosystems, Buffalo Grove, IL) using the Ventana Benchmark ULTRA automated slide processing system (Roche Diagnostics, Mannheim, Germany). Expression of p53 was scored across 170 breast cancers as overexpression (OE), complete absence (CA), cytoplasmic (CY), or wild type (WT) as described in Koebel el al. ${ }^{43}$. Normal or WT staining was characterized by variable staining intensity. Abnormal $\mathrm{OE}$ staining was strongly intense staining in $>50 \%$ tumor cell nuclei. Abnormal CA staining was the complete absence of expression within tumor cell nuclei, with a variable intensity of normal p53 expression seen in fibroblasts and lymphocytes acting as an intrinsic control. Abnormal CY staining was diffuse cytoplasmic staining in the absence of strong nuclear staining.

\section{TIL evaluation}

Stromal TILs were assessed for all cases in the highest and lowest quintile of MD using one H\&E-stained section of the whole FFPE block. The evaluation was conducted according to the standardized approach for TIL evaluation in breast cancer from the TILs working group ${ }^{44}$. In short, mononuclear stromal TILs within the borders of the invasive tumor were evaluated but excluding areas of necrosis, previous core biopsy sites, and granulocytes. The estimation was semi-quantitative, assessing an average percentage of stromal TILs in the tumor area for the full section, with no evaluation of hotspots. Independent assessment was performed by D.C. and D.B., and a consensus was reached for discordant cases.

\section{Statistical analysis}

A two-tailed $t$-test (continuous data), two-tailed Fisher's exact test $(2 \times 2$ and $3 \times 2$ tables), or chi-square test $(4 \times 2$ or greater tables) were applied to compare clinico-pathological differences between the highest and lowest quintiles of MD. MD quintiles were selected prior to the analysis conducted for this study, as done by others ${ }^{45-47}$. Mann-Whitney tests were used to calculate differences in FGA, HRD, and mutation burden between the two density cohorts. Error bars represent the standard deviation.

\section{Reporting summary}

Further information on research design is available in the Nature Research Reporting Summary linked to this article.

\section{DATA AVAILABILITY}

The DNA sequencing data generated during the study are publicly available in the NCBI Sequence Read Archive (SRA): https://identifiers.org/ncbi/insdc.sra: SRP $269052^{37}$. All other datasets generated and analyzed during the current study will be made available on reasonable request, from the corresponding author D.C., as described in the following figshare metadata record: https://doi.org/10.6084/m9. figshare.12601754 (ref. ${ }^{29}$ ).

Received: 5 January 2020; Accepted: 13 July 2020; Published online: 07 August 2020

\section{REFERENCES}

1. Boyd, N. F. et al. Heritability of mammographic density, a risk factor for breast cancer. N. Engl. J. Med. 347, 886-894 (2002). 
2. McCormack, V. A. \& dos Santos Silva, I. Breast density and parenchymal patterns as markers of breast cancer risk: a meta-analysis. Cancer Epidemiol. Biomark. Prev. 15, 1159-1169 (2006)

3. Nazari, S. S. \& Mukherjee, P. An overview of mammographic density and its association with breast cancer. Breast Cancer 25, 259-267 (2018).

4. Kavanagh, A. M. et al. Using mammographic density to improve breast cancer screening outcomes. Cancer Epidemiol. Biomark. Prev. 17, 2818-2824 (2008).

5. Nickson, C. \& Kavanagh, A. M. Tumour size at detection according to different measures of mammographic breast density. J. Med. Screen. 16, 140-146 (2009).

6. DeFilippis, R. A. et al. CD36 repression activates a multicellular stromal program shared by high mammographic density and tumor tissues. Cancer Discov. 2, 826-839 (2012).

7. Theocharis, A. D. et al. Insights into the key roles of proteoglycans in breast cancer biology and translational medicine. Biochim. Biophys. Acta 1855, 276-300 (2015).

8. Alowami, S., Troup, S., Al-Haddad, S., Kirkpatrick, I. \& Watson, P. H. Mammographic density is related to stroma and stromal proteoglycan expression. Breast Cancer Res. 5, R129-R135 (2003)

9. Li, T. et al. The association of measured breast tissue characteristics with mammographic density and other risk factors for breast cancer. Cancer Epidemiol. Biomark. Prev. 14, 343-349 (2005)

10. Yang, W. T. et al. Decreased TGFbeta signaling and increased COX2 expression in high risk women with increased mammographic breast density. Breast Cancer Res. Treat. 119, 305-314 (2010)

11. Lindström, S. et al. Genome-wide association study identifies multiple loci associated with both mammographic density and breast cancer risk. Nat. Commun. $\mathbf{5}$, 5303-5303 (2014).

12. Keller, B. M. et al. Associations between breast density and a panel of single nucleotide polymorphisms linked to breast cancer risk: a cohort study with digital mammography. BMC Cancer 15, 143 (2015).

13. Lindström, S. et al. Common variants in ZNF365 are associated with both mammographic density and breast cancer risk. Nat. Genet. 43, 185-187 (2011)

14. Gierach, G. L. et al. Mammographic density does not differ between unaffected BRCA1/2 mutation carriers and women at low-to-average risk of breast cancer. Breast Cancer Res. Treat. 123, 245-255 (2010).

15. Mitchell, G. et al. Mammographic density and breast cancer risk in BRCA1 and BRCA2 mutation carriers. Cancer Res. 66, 1866-1872 (2006).

16. Coates, A. S. et al. Tailoring therapies-improving the management of early breast cancer: St Gallen International Expert Consensus on the Primary Therapy of Early Breast Cancer 2015. Ann. Oncol. 26, 1533-1546 (2015).

17. Samreen, N., Hunt, K. N., Hruska, C. B. \& Rhodes, D. J. Molecular breast imaging detected invasive lobular carcinoma in dense breasts: a case report. Clin. Case Rep. 7, 442-444 (2019).

18. Jiang, L. et al. Mammographic features are associated with clinicopathological characteristics in invasive breast cancer. Anticancer Res. 31, 2327-2334 (2011).

19. Sun, S.-S., Zhang, B., Zhao, H.-M. \& Cao, X.-C. Association between mammographic features and clinicopathological characteristics in invasive ductal carcinoma of breast cancer. Mol. Clin. Oncol. 2, 623-629 (2014).

20. Moran, O. et al. Predictors of mammographic density among women with a strong family history of breast cancer. BMC Cancer 19, 631 (2019).

21. Martin, L. J. et al. Family history, mammographic density, and risk of breast cancer. Cancer Epidemiol. Biomark. Prev. 19, 456-463 (2010).

22. Maskarinec, G. et al. Mammographic density and breast cancer risk by family history in women of white and Asian ancestry. Cancer Causes Control 26, 621-626 (2015).

23. Huo, C. W. et al. High mammographic density in women is associated with protumor inflammation. Breast Cancer Res. 20, 92 (2018).

24. Liu, Y. et al. Deletions linked to TP53 loss drive cancer through p53-independent mechanisms. Nature 531, 471-475 (2016).

25. Miller, L. D. et al. An expression signature for p53 status in human breast cancer predicts mutation status, transcriptional effects, and patient survival. Proc. Natl Acad. Sci. USA 102, 13550-13555 (2005).

26. Ciriello, G. et al. Emerging landscape of oncogenic signatures across human cancers. Nat. Genet. 45, 1127-1133 (2013).

27. Knijnenburg, T. A. et al. Genomic and molecular landscape of DNA damage repair deficiency across The Cancer Genome Atlas. Cell Rep. 23, 239-254.e236 (2018).

28. Cheasley, D. et al. Molecular comparison of interval and screen-detected breast cancers. J. Pathol. 248, 243-252 (2019).

29. Cheasley, D. et al. The TP53 mutation rate differs in breast cancers that arise in women with high or low mammographic density. figshare. https://doi.org/ 10.6084/m9.figshare.12601754 (2020).

30. Nickson, C. et al. AutoDensity: an automated method to measure mammographic breast density that predicts breast cancer risk and screening outcomes. Breast Cancer Res. 15, R80 (2013).
31. Nickson, $\mathrm{C}$. et al. Prospective validation of the $\mathrm{NCl}$ breast cancer risk assessment tool and the autodensity mammographic density tool on 40,000 australian screening program participants. J. Glob. Oncol. 4, 49s-49s (2018).

32. Nickson, $C$. et al. AutoDensity: an automated method to measure mammographic breast density that predicts breast cancer risk and screening outcomes. Breast Cancer Res. 15, R80 (2013).

33. Pereira, B. et al. The somatic mutation profiles of 2,433 breast cancers refines their genomic and transcriptomic landscapes. Nat. Commun. 7, 11479 (2016).

34. $\mathrm{Li}, \mathrm{N}$. et al. Evaluating the breast cancer predisposition role of rare variants in genes associated with low-penetrance breast cancer risk SNPs. Breast Cancer Res. 20, 3 (2018).

35. Li, N. et al. Mutations in RECQL are not associated with breast cancer risk in an Australian population. Nat. Genet. https://doi.org/10.1038/s41588-018-0206-9 (2018).

36. Rowley, S. M. et al. Population-based genetic testing of asymptomatic women for breast and ovarian cancer susceptibility. Genet. Med. https://doi.org/10.1038/ s41436-018-0277-0 (2018).

37. Cheasley, D. et al. The TP53 mutation rate differs in breast cancers that arise in women with high or low mammographic density. NCBI Sequence Read Archive. https://identifiers.org/ncbi/insdc.sra:SRP269052 (2020).

38. Kuilman, T. et al. CopywriteR: DNA copy number detection from off-target sequence data. Genome Biol. 16, 49 (2015).

39. Marquard, A. M. et al. Pan-cancer analysis of genomic scar signatures associated with homologous recombination deficiency suggests novel indications for existing cancer drugs. Biomark. Res. 3, 9 (2015).

40. Popova, T. et al. Ploidy and large-scale genomic instability consistently identify basal-like breast carcinomas with BRCA1/2 inactivation. Cancer Res. 72 5454-5462 (2012).

41. Abkevich, V. et al. Patterns of genomic loss of heterozygosity predict homologous recombination repair defects in epithelial ovarian cancer. Br. J. Cancer 107 1776-1782 (2012).

42. Burrell, R. A. et al. Replication stress links structural and numerical cancer chromosomal instability. Nature 494, 492-496 (2013).

43. Köbel, M. et al. Optimized p53 immunohistochemistry is an accurate predictor of TP53 mutation in ovarian carcinoma. J. Pathol. Clin. Res. 2, 247-258 (2016).

44. Salgado, R. et al. The evaluation of tumor-infiltrating lymphocytes (TILs) in breast cancer: recommendations by an International TILs Working Group 2014. Ann. Oncol. 26, 259-271 (2015).

45. Astley, S. M. et al. A comparison of five methods of measuring mammographic density: a case-control study. Breast Cancer Res. 20, 10 (2018).

46. Habel, L. A. et al. Case-control study of mammographic density and breast cancer risk using processed digital mammograms. Breast Cancer Res. 18, 53 (2016).

47. Shepherd, J. A. et al. Volume of mammographic density and risk of breast cancer Cancer Epidemiol. Biomark. Prev. 20, 1473-1482 (2011).

\section{ACKNOWLEDGEMENTS}

The authors would like to acknowledge the Bioinformatics and Molecular Genomics Core Facilities of the Peter MacCallum Cancer Centre. This work would not have been possible without financial support by the Victorian Cancer Agency (ECSG15003, to D.C.), the National Breast Cancer Foundation (IF-15-004 \& CG-08-02, to I.G.C.), the NHMRC (APP1079329 to S.B.F.), and Cancer Australia (1084963, to I.G.C.). The funders had no role in the design and execution of this study.

\section{AUTHOR CONTRIBUTIONS}

S.B.F. and D.B. performed pathology review of cases; D.B. prepared TMAs and performed IHC; D.C., S.H., and H.S. prepared tissue samples, performed nucleic acid extraction, and sequencing library preparations; C.N., P.P., G.L., and V.P. provided MD information; L.D., K.E., G.B.M., and S.B.F. provided patient clinical information; D.C., L.D., S.H., G.L., K.E., S.M.R., H.S., and K.M.F. recorded clinico-pathological information; D.C., S.H., T.K., and B.L. performed bioinformatics analyses; D.C. and D.B. performed TILs assessment; L.D. and I.G.C. coordinated the Lifepool study; D.C., L.D., S.H., P.P., G.L., K.L.G., and I.G.C. analyzed the data; D.C., K.L.G., and I.G.C. designed the study and were involved at all stages; D.C. prepared the figures and drafted the final manuscript, which was then extensively edited by all remaining authors.

\section{COMPETING INTERESTS}

The authors declare no competing interests. 


\section{ADDITIONAL INFORMATION}

Supplementary information is available for this paper at https://doi.org/10.1038/ s41523-020-00176-7.

Correspondence and requests for materials should be addressed to D.C.

Reprints and permission information is available at http://www.nature.com/ reprints

Publisher's note Springer Nature remains neutral with regard to jurisdictional claims in published maps and institutional affiliations.
Open Access This article is licensed under a Creative Commons Attribution 4.0 International License, which permits use, sharing, adaptation, distribution and reproduction in any medium or format, as long as you give appropriate credit to the original author(s) and the source, provide a link to the Creative Commons license, and indicate if changes were made. The images or other third party material in this article are included in the article's Creative Commons license, unless indicated otherwise in a credit line to the material. If material is not included in the article's Creative Commons license and your intended use is not permitted by statutory regulation or exceeds the permitted use, you will need to obtain permission directly from the copyright holder. To view a copy of this license, visit http://creativecommons. org/licenses/by/4.0/.

(c) The Author(s) 2020 